J. H. Keum

Nagoya Math. J.

Vol. 118 (1990), 99-110

\title{
EVERY ALGEBRAIC KUMMER SURFACE IS THE K3-COVER OF AN ENRIQUES SURFACE
}

\author{
JONG HAE KEUM
}

\section{Introduction}

A Kummer surface is the minimal desingularization of the surface $T / i$, where $T$ is a complex torus of dimension 2 and $i$ the involution automorphism on $T$. $T$ is an abelian surface if and only if its associated Kummer surface is algebraic. Kummer surfaces are among classical examples of K3-surfaces (which are simply-connected smooth surfaces with a nowhere-vanishing holomorphic 2-form), and play a crucial role in the theory of K3-surfaces. In a sense, all Kummer surfaces (resp. algebraic Kummer surfaces) form a 4 (resp. 3)-dimensional subset in the 20 (resp. 19)-dimensional family of K3-surfaces (resp. algebraic K3 surfaces).

An Enriques surface is a smooth projective surface $Y$ with $2 K_{Y}=0$, $H^{1}\left(Y, \mathcal{O}_{Y}\right)=H^{2}\left(Y, \mathcal{O}_{Y}\right)=0$. The unramified double cover of $Y$ defined by the torsion class $K_{Y}$ is an algebraic K3-surface. Conversely, if an algebraic K3-surface $X$ admits a fixed-point-free involution $\tau$, then the quotient surface $X / \tau$ is an Enriques surface. It is known that all Enriques surfaces form a 10-dimensional moduli space.

Let $X$ be a surface. The Picard number of $X$, denoted by $\rho(X)$, is the rank of the Neron-Severi group $\mathrm{NS}(X)$, the sublattice of $H^{2}(X, Z)$ generated by algebraic cycles. The transcendental lattice $T_{X}$ of $X$ is the orthogonal complement of $\mathrm{NS}(X)$ in $H^{2}(X, Z)$. If $X$ is a K3-surface, then $0 \leq \rho(X) \leq 20$. If $X$ is the K3-cover of an Enriques surface, then $\rho(X)$ $\geq 10$.

Let $L$ be a lattice, i.e. a free $Z$-module of finite rank together with a $Z$-valued symmetric bilinear form. For every integer $m$ we denote by $L(m)$ the lattice obtained from $L$ by multiplying the values of its bilinear form by $m$. The length of $L$, denoted by $l(L)$, is the minimum number of generators of $L^{*} / j(L)$, where $j: L \rightarrow L^{*}=\operatorname{Hom}(L, Z)$ is the natural

Received November 28, 1988. 
homomorphism.

Let $U$ and $E_{8}$ denote the even unimodular lattices of signature $(1,1)$ and $(0,8)$ respectively.

Theorem 1. (Criterion for a K3-surface to cover an Enriques surface). Let $X$ be an algebraic K3-surface.

Assume that $l\left(T_{X}\right)+2 \leq \rho(X)$. (This is true if $\rho(X) \geq 12$.)

Then the following are equivalent.

(i) X admits a fixed-point-free involution.

(ii) There exists a primitive embedding of $T_{X}$ into $\Lambda^{-}=U \oplus U(2) \oplus$ $E_{8}(2)$ such that the orthogonal complement of $T_{X}$ in $A^{-}$contains no vectors of self-intersection -2 .

Remark. The assumption that $l\left(T_{X}\right)+2 \leq \rho(X)$ is needed only for the part (ii) $\Rightarrow$ (i).

Theorem 1 is a consequence of the uniqueness theorem on embeddings of even lattices [Nik 2] and the "surjectivity of the period map for Enriques surfaces" [Ho 2]. Making use of Theorem 1 and the criterion, due to Nikulin, for a K3-surface to be Kummer ([Nik 1] \& [Mor]) we can prove the following result:

THEOREM 2. Every algebraic Kummer surface is the K3-cover of some Enriques surface.

\section{§ 0. Preliminaries}

(0.1) Definition. A $Z$-module isomorphism of lattices preserving the bilinear form is called an isometry. The group of self-isometries of a lattice $L$, denoted by $O(L)$, is called the orthogonal group of $L$ (or the group of units).

A lattice is even if the associated quadratic form takes on only even integer values, and is odd if the quadratic form takes on some odd value.

The discriminant of a lattice $L$, written $\operatorname{discr}(L)$, is the determinant of the matrix of its bilinear form. A lattice is non-degenerate if its discriminant is non-zero, and unimodular if its discriminant is \pm 1 . If $L$ is a non-degenerate lattice, the signature of $L$ is a pair $\left(t_{+}, t_{-}\right)$, where $t_{ \pm}$ denotes the multiplicity of the eigenvalue \pm 1 for the quadratic form on $L \otimes R$. A lattice is indefinite if the associated quadratic form takes on both positive and negative values. An indefinite lattice $L$ of signature $\left(1, t_{-}\right)$or $\left(t_{+}, 1\right)$ is called a hyperbolic lattice. 
An embedding $L \rightarrow M$ is primitive if $M / L$ is torsion free.

(0.2) Examples. (i) By $A_{n}, D_{n}, E_{n}$ we denote the even negative definite lattices defined by the matrix equal to the Cartan matrix of an irreducible root system of type $A_{n}, D_{n}, E_{n}$ respectively [Bour; Chap. VI]. For example, the bilinear form on $E_{8}$ is given by the matrix

$$
\left(\begin{array}{rrrrrrrr}
-2 & 1 & 0 & 0 & 0 & 0 & 0 & 0 \\
1 & -2 & 1 & 0 & 0 & 0 & 0 & 0 \\
0 & 1 & -2 & 1 & 1 & 0 & 0 & 0 \\
0 & 0 & 1 & -2 & 0 & 0 & 0 & 0 \\
0 & 0 & 1 & 0 & -2 & 1 & 0 & 0 \\
0 & 0 & 0 & 0 & 1 & -2 & 1 & 0 \\
0 & 0 & 0 & 0 & 0 & 1 & -2 & 1 \\
0 & 0 & 0 & 0 & 0 & 0 & 1 & -2
\end{array}\right) .
$$

$E_{8}$ is the only unimodular lattice among $A_{n}, D_{n}, E_{n}$ 's.

(ii) $U$ denotes the hyperbolic lattice of rank 2 defined by the matrix

$$
\left(\begin{array}{ll}
0 & 1 \\
1 & 0
\end{array}\right)
$$

This is an even unimodular lattice; note that $U(-m) \cong U(m)$ for any integer $m$.

(iii) For any integer $n$ we denote by $\langle n\rangle$ the lattice $Z e$ with $\langle e, e\rangle$ $=n$.

(0.3) Theorem [Nik 2; Corollary 1.12.3 \& Theorem 1.14.4]. A primitive embedding of an even non-degenerate lattice $L$ of signature $\left(s_{+}, s_{-}\right)$into an even unimodular lattice $M$ of signature $\left(t_{+}, t_{-}\right)$exists provided that

$$
s_{+} \leq t_{+}, \quad s_{-} \leq t_{-}, \quad \text { and } \quad l(L)+1 \leq \operatorname{rank}(M)-\operatorname{rank}(L) .
$$

Furthermore, if the three inequalities are all strict, then the primitive embedding is unique.

The following corollaries will be used later.

(0.3.1) Corollary. There is a primitive embedding of $\langle-2\rangle \oplus\langle-2 m\rangle$ into the even unimodular lattice $E_{8}$ for any positive integer $m$.

Proof. Note that $\operatorname{sign}\left(E_{8}\right)=(0,8)$, sign $(\langle-2\rangle \oplus\langle-2 m\rangle)=(0,2)$, and $l(\langle-2\rangle \oplus\langle-2 m\rangle)=2$. The corollary follows from $(0.3)$ Q.E.D. 
(0.3.2) Conollary. There is a primitive embedding of $\langle-2 m\rangle$ into $E_{8}$ for any positive integer $m$.

(0.4) Let $X$ be a K3-surface. It is known (see [B-P-V; Chap. VIII]) that $H^{2}(X, Z)$ (with its intersection pairing) is isomorphic as an abstract lattice to the lattice, called the K3-lattice,

$$
\Lambda=U \oplus U \oplus U \oplus E_{8} \oplus E_{8} .
$$

(0.5) Definition. Let $T$ be a complex torus of dimension two. The involution automorphism $i: T \rightarrow T, i(x)=-x$, has sixteen fixed points, namely the points of order 2 on $T$. The quotient surface $T / i$ has sixteen ordinary double points (i.e. singular points of type $A_{1}$ ). Resolving the double points we obtain a smooth surface $X$, called the Kummer surface of $T$. $X$ has sixteen exceptional curves of self-intersection -2 arising from the resolution of singularities. Note that $T$ is an abelian surface if and only if its associated Kummer surface is an algebraic K3-surface.

(0.6) THEOREM [Nik 1]. There is an even, negative definite lattice II of rank 16, called the Kummer lattice, with the following properties:

(i) $\Pi$ admits a unique primitive embedding into the $K 3$ lattice $A$ and its orthogonal complement in $\Lambda$ is isomorphic to $U(2)^{3}$.

(ii) If $X$ is a Kummer surface, then the minimal primitive sublattice of $H^{2}(X, Z)$ containing the classes of the sixteen exceptional curves on $X$ is isomorphic to $I I$.

(iii) A K3-surface $X$ is Kummer if and only if there is a primitive embedding of $\Pi$ into the Néron-Severi group $\mathrm{NS}(X)$.

The following criterion is an alternative to (0.6) (iii).

(0.6.1) CoRollary [Mor; Cor. 4.4]. Let $X$ be an algebraic K3-surface.

(i) If $\rho(X)=19$ or 20 , then $X$ is a Kummer surface if and only if there is an even lattice $T^{\prime}$ with $T_{X} \cong T^{\prime}(2)$.

(ii) If $\rho(X)=18$, then $X$ is a Kummer surface if and only if there is an even lattice $T^{\prime}$ with $T_{X} \cong U(2) \oplus T^{\prime}(2)$.

(iii) If $\rho(X)=17$, then $X$ is a Kummer surface if and only if there is an even lattice $T^{\prime}$ with $T_{X} \cong U(2)^{2} \oplus T^{\prime}(2)$.

(iv) If $\rho(X) \leq 16$, then $X$ is not a Kummer surface.

\section{$\S 1$. Proof of Theorem 1}

(1.1) Let $X$ be a K3 surface. Since $p_{g}(X)=1$, the choice of an 
isometry $\phi: H^{2}(X, Z) \rightarrow \Lambda$ determines a line in $\Lambda_{C}=\Lambda \otimes C$ spanned by the $\phi_{C}$-image of a nowhere vanishing holomorphic 2 -form $\omega_{X}$. The point $\left[\omega_{X}\right] \in \boldsymbol{P}\left(\Lambda_{C}\right)$ is called the period point of the marked K3-surface $(X, \phi)$.

The following theorem goes by the name "(weak) Torelli theorem for K3 surfaces".

(1.2) Theorem ([P-S], [B-R], [B-P-V]). Two K3 surfaces are isomorphic if and only if there are markings for them, such that the corresponding period points are the same.

(1.3) Let $Y$ be an Enriques surface. It is known (see [B-P-V; Chap. VIII]) that

$$
\operatorname{Pic}(Y) \cong \mathrm{NS}(Y) \cong H^{2}(Y, Z) \cong Z^{10} \oplus Z / 2 Z
$$

and that the lattice $H^{2}(Y, Z)_{f}$, the torsion-free-part of $H^{2}(Y, Z)$, is isomorphic to the even unimodular lattice $U \oplus E_{8}$ of signature $(1,9)$. If $X$ is the K3-cover of $Y$, then $\operatorname{Pic}(X)$ contains $p^{*}(\operatorname{Pic}(Y)) \cong U(2) \oplus E_{8}(2)$ as a primitive sublattice, where $p: X \rightarrow Y$ is the covering projection. In particular, $\rho(X) \geq 10$.

(1.4) Let $\Lambda$ be the K3-lattice, that is,

$$
\Lambda=U \oplus U \oplus U \oplus E_{8} \oplus E_{8} .
$$

We fix a basis of $\Lambda$ of the form $v_{1}, v_{2}, v_{1}^{\prime}, v_{2}^{\prime}, v_{1}^{\prime \prime}, v_{2}^{\prime \prime}, e_{1}^{\prime}, \cdots, e_{8}^{\prime}, e_{1}^{\prime \prime}, \cdots, e_{8}^{\prime \prime}$, where the first three pairs are the standard bases of $U$ and the remaining two octuples are the standard bases of $E_{8}$.

Let $\theta: \Lambda \rightarrow \Lambda$ be the involution given by the formula

$$
\begin{aligned}
& \theta\left(v_{i}\right)=-v_{i}, \quad \theta\left(v_{i}^{\prime}\right)=v_{i}^{\prime \prime}, \quad \theta\left(v_{i}^{\prime \prime}\right)=v_{i}^{\prime}, \quad i=1,2, \\
& \theta\left(e_{i}^{\prime}\right)=e_{i}^{\prime \prime}, \quad \theta\left(e_{i}^{\prime \prime}\right)=e_{i}^{\prime}, \quad i=1, \cdots, 8 .
\end{aligned}
$$

Then the $\theta$-invariant sublattice, denoted by $\Lambda^{+}$, is

$$
\Lambda^{+}=Z y_{1} \oplus Z y_{2} \oplus Z e_{1} \oplus \cdots \oplus Z e_{8},
$$

where $y_{i}=v_{i}^{\prime}+v_{i}^{\prime \prime}, i=1,2$ and $e_{i}=e_{i}^{\prime}+e_{i}^{\prime \prime}, i=1, \cdots, 8$.

The $\theta$-anti-invariant sublattice, denoted by $\Lambda^{-}$, is

$$
\Lambda^{-}=Z \bar{y}_{1} \oplus Z \bar{y}_{2} \oplus \boldsymbol{Z} \bar{e}_{1} \oplus \cdots \oplus \boldsymbol{Z} \bar{e}_{8}^{\prime} \oplus \boldsymbol{Z} v_{1} \oplus \boldsymbol{Z} v_{2},
$$

where $\bar{y}_{i}=v_{i}^{\prime}-v_{i}^{\prime \prime}, i=1,2, \bar{e}_{i}=e_{i}^{\prime}-e_{i}^{\prime \prime}, i=1, \cdots, 8$.

It is easy to see that 


$$
\Lambda^{+} \cong U(2) \oplus E_{8}(2), \quad \Lambda^{-} \cong U(2) \oplus E_{8}(2) \oplus U, \quad\left(\Lambda^{+}\right)^{\perp}=\Lambda^{-},
$$

and that both $\Lambda^{-}$and $\Lambda^{-}$are primitive sublattices of $\Lambda$.

(1.5) Lemma [Ho 1; Theorem 5.1]. Let $X$ be the K3-cover of an Enriques surface $Y$, and let $\tau: X \rightarrow X$ be the covering involution.

Then there exists an isometry

$$
\phi: H^{2}(X, Z) \longrightarrow \Lambda
$$

such that the following diagram

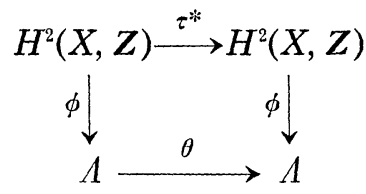

commutes.

In particular, $\phi$ induces an isomorphism

$$
\bar{\phi}: H^{2}(X, Z)^{z^{*}}=p^{*} H^{2}(Y, Z)=p^{*} \operatorname{Pic}(Y) \longrightarrow \Lambda^{+},
$$

where $p: X \rightarrow Y$ is the covering projection.

(1.6) Remark. The choice of $\phi$ as in Lemma (1.5) is unique up to

$$
\Gamma=\{g \in O(\Lambda): g \circ \theta=\theta \circ g\} .
$$

(1.7) A marked Enriques surface is a pair $(Y, \phi)$ with $Y$ an Enriques surface and $\phi: H^{2}(X, Z) \rightarrow \Lambda$ an isometry satisfying $\phi \circ \tau^{*}=\theta \circ \phi$, as in Lemma (1.5). Since $\tau^{*} \omega_{X}=-\omega_{X}$ (there is no holomorphic 2-form on $Y$ ), the period point $\left[\omega_{X}\right]$, called the period point of $(Y, \phi)$, of the marked $\mathrm{K} 3$ surface $(X, \phi)$ belongs to the set

$$
\left.\Omega^{-}=\left\{[\omega] \in \boldsymbol{P}\left(\Lambda^{-} \otimes \boldsymbol{C}\right):\langle\omega, \omega\rangle=0,\langle\omega, \bar{\omega}\rangle\right\rangle 0\right\} .
$$

By Remark (1.6), the assignment

$$
Y: \longrightarrow\left[\omega_{X}\right] \in \Omega^{-} / \Gamma_{r},
$$

where $\Gamma_{r}=\left\{\left.g\right|_{A^{-}}: g \in \Gamma\right\}$, is well defined and called the period map for Enriques surfaces.

Global Torelli theorem for Enriques surfaces [Ho 1] says that this period map is injective.

(1.8) Notation. 


$$
\begin{aligned}
& \Omega_{0}^{-}=\left\{[\omega] \in \Omega^{-}:\langle\omega, \delta\rangle \neq 0 \text { for any } \delta \in \Lambda^{-},\langle\delta, \delta\rangle=-2\right\} \\
& D_{0}=\Omega_{0}^{-} / \Gamma_{r} .
\end{aligned}
$$

The next theorem, which is due to Horikawa, goes by the name "the surjectivity of the period map for Enriques surfaces".

(1.9) Theorem ([Ho 2] \& [B-P-V]). Every point of $D_{0}$ is the period point of an Enriques surface. In other words, every point of $\Omega_{0}^{-}$is the period point of some marked Enriques surface.

(1.10) Lemma. Let $X$ be a K3 surface, and let $d$ be a divisor on $X$ of self-intersection $\geq-2$. Then either $d$ or $-d$ is effective.

Proof. Riemann-Roch yields the inequality

$$
h^{0}\left(\mathcal{O}_{X}(d)\right)+h^{0}\left(\mathcal{O}_{X}(-d)\right)=\frac{1}{2}\langle d, d\rangle+2+h^{1}\left(\mathcal{O}_{X}(-d)\right) \geq 1,
$$

and hence $\mathcal{O}_{X}(d)$ or $\mathcal{O}_{X}(-d)$ has a non-trivial section.

Q.E.D.

(1.11) Proof of Theorem 1.

(i) $\Rightarrow$ (ii): Let $p: X \rightarrow Y$ be the unramified covering of an Enriques surface $Y$, and let $\tau, \phi$ be the same as in (1.5).

Then, $\phi(\operatorname{Pic}(X)) \supseteq \phi\left(p^{*}(\operatorname{Pic}(Y))\right)=\Lambda^{+}$, so we have $\phi\left(T_{X}\right) \subseteq \Lambda^{-}$. This embedding is primitive, for an isometry preserves primitivity. Now suppose that $\Lambda^{-}$contains a vector $v$ with $v^{2}=-2, v_{\perp} \phi(T)$. Then the class $d=$ $\phi^{-1}(v)$ belongs to $\operatorname{Pic}(X)$ and, by (1.10), $d$ or $-d$ is effective. But no effective class can be $\tau^{*}$-anti-invariant as is $\pm d$.

(ii) $\Rightarrow\left(\right.$ i): Let $\psi_{1}: T_{X} \rightarrow \Lambda^{-}$be a primitive embedding such that no (-2)-vector in $\Lambda^{-}$is orthogonal to $\psi_{1}\left(T_{X}\right)$.

Claim. $\psi_{1}$ extends to an isometry $\psi: H^{2}(X, Z) \rightarrow \Lambda$.

Proof of the claim. Fix an isometry $\psi_{2}: H^{2}(X, Z) \rightarrow \Lambda$.

Then we have two embeddings of $T=T_{X}$ into $\Lambda$, namely,

$$
\psi_{1}: T \longrightarrow \Lambda^{-} \subseteq \Lambda \text { and }\left.\psi_{2}\right|_{T}: T \longrightarrow \Lambda \text {. }
$$

Since $T$ has signature $(2,20-\rho)$ and since $l(T)+2 \leq \rho(X)=\operatorname{rank}(\Lambda)-$ $\operatorname{rank}(T)$, by $(0.3)$, there exists $\nu \in O(\Lambda)$ such that $\psi_{1}=\left.\nu \circ \psi_{2}\right|_{T}$. We take $\psi=\nu \circ \psi_{2}$ and the claim is proved. Now, since $\omega_{X} \in T \otimes C \subseteq H^{2}(X, C)$, the period point $\left[\omega_{X}\right]$ of the marked K3-surface $(X, \psi)$ belongs to $\Omega_{0}^{-}$. By (1.9), there exists a marked Enriques surface $(Y, \phi)$ whose period point is equal to $\left[\omega_{X}\right]$. But then, by (1.2), the K3-cover $X^{\prime}$ of $Y$ is isomorphic 
to $X$, and the covering involution on $X^{\prime}$ lifts to a fixed-point-free involution on $X$.

Q.E.D.

\section{§ 2. Proof of Theorem 2}

By Theorem 1, it suffices to prove that there exists a primitive embedding of $T_{X}$ into $\Lambda^{-}$with no (-2)-vectors in the orthogonal complement. We split the proof into four cases.

Case 1. $\rho(X)=17$.

By (0.6.1) (iii), $T_{x}=U(2) \oplus U(2) \oplus\langle-4 m\rangle$ for some positive integer $m$. There exists such a primitive embedding $T_{X} \rightarrow \Lambda^{-}=U(2) \oplus U \oplus E_{8}(2)$ if there exists a primitive embedding $U(2) \oplus\langle-4 m\rangle \rightarrow U \oplus E_{8}(2)$ with no $(-2)$-vectors in the orthogonal complement.

Let $\{\boldsymbol{x}, \boldsymbol{y}, \boldsymbol{t}\}$ be a standard basis of $U(2) \oplus\langle-4 m\rangle$, that is

$$
x^{2}=y^{2}=\langle\boldsymbol{x}, \boldsymbol{t}\rangle=\langle\boldsymbol{y}, \boldsymbol{t}\rangle=0,\langle\boldsymbol{x}, \boldsymbol{y}\rangle=2, \quad \boldsymbol{t}^{2}=-4 m,
$$

and let $\{\boldsymbol{e}, \boldsymbol{f}\}$ be a standard basis of $U$,

$$
e^{2}=f^{2}=0, \quad\langle e, f\rangle=1,
$$

By (0.3.1), we can pick up two elements $\boldsymbol{w}_{1}$ and $\boldsymbol{w}_{2}$ of $E_{8}(2)$ which generate a primitive sublattice of $E_{8}(2)$ isomorphic to $\langle-4\rangle \oplus\langle-4 m\rangle$. Define a map $\phi: U(2) \oplus\langle-4 m\rangle \rightarrow U \oplus E_{8}(2)$ by the formula

$$
\begin{aligned}
& \phi(x)=e, \\
& \phi(y)=e+2 f+w_{1}, \\
& \phi(t)=w_{2} .
\end{aligned}
$$

Then, $\phi(\boldsymbol{x})^{2}=\phi(\boldsymbol{y})^{2}=\langle\phi(\boldsymbol{x}), \phi(\boldsymbol{t})\rangle=\langle\phi(\boldsymbol{y}), \phi(\boldsymbol{t})\rangle=0$,

$$
\langle\phi(x), \phi(y)\rangle=2 \text { and } \phi(t)^{2}=-4 m \text {. }
$$

So, $\phi$ is an embedding.

If $k d, d \in U \oplus E_{8}(2), k \in Z$, belongs to $\operatorname{im} \phi$, then $k \boldsymbol{d}=h \boldsymbol{e}+i\left(\boldsymbol{e}+2 \boldsymbol{f}+\boldsymbol{w}_{1}\right)+j \boldsymbol{w}_{2}$ for some $h, i, j \in \boldsymbol{Z}$.

Write $d=u+w, u \in U, w \in E_{8}(2)$, then

$$
\begin{aligned}
& k \boldsymbol{u}=(h+i) \boldsymbol{e}+2 i \boldsymbol{f}, \\
& k \boldsymbol{w}=i \boldsymbol{w}_{1}+j \boldsymbol{w}_{2} .
\end{aligned}
$$

Since $\left\{\boldsymbol{w}_{1}, \boldsymbol{w}_{2}\right\}$ generates a primitive sublattice of $E_{8}(2), k$ divides both $i$ and $j$ and hence $h$. Thus $d \in \operatorname{im} \phi$. This proves that $\phi$ is primitive. 
If $\boldsymbol{d} \in U \oplus E_{8}(2)$ is orthogonal to im $\phi$, then $\boldsymbol{d}$ has to be of the form

$$
\boldsymbol{d}=k \boldsymbol{e}+\boldsymbol{w}, \quad k \in Z, \quad \boldsymbol{w} \in E_{8}(2) .
$$

But then $\boldsymbol{d}^{2}=\boldsymbol{w}^{2} \neq-2$, for $E_{8}$ is even. Therefore, the orthogonal complement of $\operatorname{im} \phi$ contains no $(-2)$-vectors.

Case 2. $\rho(X)=18$.

By $(0.6 .1)$ (ii), $T_{X} \cong U(2) \oplus T^{\prime}(2)$ for some even lattice $T^{\prime}$ of signature $(1,1)$. Since $T^{\prime}(2)$ is indefinite, there is a primitive element $y$ of $T^{\prime}(2)$, $\langle\boldsymbol{y}, \boldsymbol{y}\rangle<0$. Extend $\{\boldsymbol{y}\}$ to a basis $\{\boldsymbol{x}, \boldsymbol{y}\}$ of $T^{\prime}(2)$. Then $\boldsymbol{x}^{2}=4 a, \boldsymbol{y}^{2}=4 c$, $\langle\boldsymbol{x}, \boldsymbol{y}\rangle=2 b$ for some integers $a, b$ and $c, c<0$.

By (0.3.2), one can pick up a primitive element $w, w^{2}=4 c$, of $E_{8}(2)$. Define a map $\psi: T^{\prime}(2) \rightarrow U \oplus E_{8}(2)$ by the formula

$$
\begin{aligned}
& \psi(\boldsymbol{x})=\boldsymbol{e}+2 a \boldsymbol{f} \\
& \psi(\boldsymbol{y})=2 b \boldsymbol{f}+\boldsymbol{w}
\end{aligned}
$$

where $\{\boldsymbol{e}, \boldsymbol{f}\}$ is the standard basis of $U$. Then $\psi$ is an embedding.

If $k \boldsymbol{d}, \boldsymbol{d}=\boldsymbol{u}+\boldsymbol{v}, \boldsymbol{u} \in U, \boldsymbol{v} \in E_{8}(2), k \in Z$, belongs to $\operatorname{im} \psi$, then

$$
k \boldsymbol{d}=k(\boldsymbol{u}+\boldsymbol{v})=i(\boldsymbol{e}+2 a \boldsymbol{f})+j(2 b \boldsymbol{f}+\boldsymbol{w}) .
$$

Comparing both sides, we get

$$
\begin{aligned}
& k \boldsymbol{u}=i \boldsymbol{e}+(2 a i+2 b j) \boldsymbol{f}, \\
& k \boldsymbol{v}=j \boldsymbol{w} .
\end{aligned}
$$

The primivitity of $\boldsymbol{w}$ implies that $k$ divides $j$, and hence $i$. But then $\boldsymbol{d} \in \operatorname{im} \psi$. So, $\psi$ is primitive.

If $\boldsymbol{d}=m \boldsymbol{e}+n \boldsymbol{f}+\boldsymbol{v}, m, n \in \boldsymbol{Z}, \boldsymbol{v} \in E_{8}(2)$, is orthogonal to im $\psi$, then $0=\langle\boldsymbol{d}, \psi(\boldsymbol{x})\rangle=2 a m+n$, so $n$ is an even integer. Since $E_{8}$ is even, $d^{2}=2 m n+\boldsymbol{v}^{2} \neq-2$. This proves that no $(-2)$-vector lies in the orthogonal complement of $\operatorname{im} \psi$.

Case 3. $\rho(X)=19$.

By $(0.6 .1)(\mathrm{i})$,

$$
T_{X} \cong\left(\begin{array}{ccc}
4 a & 2 d & 2 e \\
2 d & 4 b & 2 f \\
2 e & 2 f & 4 c
\end{array}\right)
$$

i.e., there exists a basis $\{\boldsymbol{x}, \boldsymbol{y}, \boldsymbol{z}\}$ of $T_{X}$ such that 


$$
\boldsymbol{x}^{2}=4 a, \quad \boldsymbol{y}^{2}=4 b, \quad \boldsymbol{z}^{2}=4 c,\langle\boldsymbol{x}, \boldsymbol{y}\rangle=2 d, \cdots, \text { etc. }
$$

Since $T_{X}$ is indefinite, we may assume $c<0$.

Let $\{\boldsymbol{e}, \boldsymbol{f}\}$ and $\{\boldsymbol{h}, \boldsymbol{k}\}$ be the standard bases of $U$ and $U(2)$, respectively. Let $w$ be the same as in Case 2, that is, $w$ is a primitive element of $E_{8}(2)$ with $\boldsymbol{w}^{2}=4 c$.

Define $\eta: T_{X} \rightarrow U \oplus U(2) \oplus E_{8}(2)$ by the formula

$$
\begin{aligned}
& \eta(\boldsymbol{x})=\boldsymbol{e}+2 a \boldsymbol{f} \\
& \eta(\boldsymbol{y})=2 d \boldsymbol{f}+\boldsymbol{h}+b \boldsymbol{k} \\
& \eta(\boldsymbol{z})=2 e \boldsymbol{f}+f \boldsymbol{k}+\boldsymbol{w} .
\end{aligned}
$$

Then $\eta$ is an embedding.

By the same argument as in Case $1 \& 2$, the primitivities of $\boldsymbol{w}, \boldsymbol{h}, \boldsymbol{e}$ imply the primitivity of $\eta$.

If $\boldsymbol{d}=i \boldsymbol{e}+j \boldsymbol{k}+k \boldsymbol{h}+m \boldsymbol{k}+\boldsymbol{v}, i, j, k, m \in \boldsymbol{Z}, \boldsymbol{v} \in E_{8}(2)$, belongs to the orthogonal complement of $\eta\left(T_{X}\right)$, then $0=\langle\boldsymbol{d}, \eta(\boldsymbol{x})\rangle=2 a i+j$, so $j$ is an even integer. But then $d^{2}=2 i j+4 k m+v^{2} \neq-2$. This proves the case 3 .

Case 4. $\rho(X)=20$.

Again, by (0.6.1) (i), $T_{X}$ has a basis $\{\boldsymbol{x}, \boldsymbol{y}\}$ with

$$
\boldsymbol{x}^{2}=4 a, \quad \boldsymbol{y}^{2}=4 c,\langle\boldsymbol{x}, \boldsymbol{y}\rangle=2 b .
$$

Define $\nu: T_{X} \rightarrow U \oplus U(2) \oplus E_{8}(2)$ by the formula

$$
\begin{aligned}
& \nu(\boldsymbol{x})=\boldsymbol{e}+2 a \boldsymbol{f} \\
& \nu(\boldsymbol{y})=2 b \boldsymbol{f}+\boldsymbol{h}+c \boldsymbol{k},
\end{aligned}
$$

where, again, $\{\boldsymbol{e}, \boldsymbol{f}\}$ and $\{\boldsymbol{h}, \boldsymbol{k}\}$ are the standard bases of $U$ and $U(2)$, respectively. Then it is easy to see that $\nu$ is a primitive embedding. It is also easy, by the same argument as in Case 3, to see that there are no (-2)-vectors in the orthogonal complement.

Q.E.D.

\section{§3. Examples}

(3.1) (Lieberman). Let $A$ be the product of two elliptic curves $E_{1}$ and $E_{2}$ and let $\left(e_{1}, e_{2}\right), e_{i} \in E_{i}, e_{i} \neq 0, i=1,2$, be a 2 -torsion point of $A$. Then the endomorphism $\sigma: A \rightarrow A$ given by the formula

$$
\sigma\left(z_{1}, z_{2}\right)=\left(-z_{1}+e_{1}, z_{2}+e_{2}\right), \quad\left(z_{1}, z_{2}\right) \in A=E_{1} \times E_{2},
$$


induces a fixed-point-free involution on the Kummer surface $\mathrm{Km}(A)$.

(3.2) Remark. Let $A$ be an abelian surface.

If $A$ splits, i.e., $A=E_{1} \times E_{2}$, a product of two elliptic curves, then $\rho(\operatorname{Km}(A)) \geq 18$. Indeed,

$\rho(A)=4$ if $E_{1}$ is isogeneous to $E_{2}$ and has a complex multiplication,

$=3$ if $E_{1}$ is isogeneous to $E_{2}$ but does not have a complex multiplication,

$=2$ if $E_{1}$ is not 1sogeneous to $E_{2}$ (cf. [Mum]).

If $\rho(A)=4$ (i.e. $\rho(\mathrm{Km}(A))=20$ ), then $A$ always splits [S-M].

If $\rho(A) \leq 3$, then $A$ may not.

(3.3) Let $A$ be a principally polarized abelian surface which does not split. Then $A$ is the Jacobian $J(C)$ of some curve $C$ of genus 2 and $\mathrm{Km}(A)$ is isomorphic to the resolution of a quartic surface $F$ in $\boldsymbol{P}^{3}$ with sixteen nodes. The equation of $F$ referred to a Göpel tetrad of nodes has the form

$$
\begin{gathered}
A\left(x^{2} t^{2}+y^{2} z^{2}\right)+B\left(y^{2} t^{2}+z^{2} x^{2}\right)+C\left(z^{2} t^{2}+x^{2} y^{2}\right) \\
=D x y z t+F(y t+z x)(z t+x y)+G(z t+x y)(x t+y z) \\
+H(x t+y z)(y t+z x)=0 \quad[\text { Hut }] .
\end{gathered}
$$

For a generic choice of coefficients, the standard Cremona transformation acts freely.

\section{REFERENCES}

[Bour] Bourbaki, N., Groupes et Algebrès de Lie, Chap. IV, V, VI., Paris, Hermann, 1968.

[B-P-V] Barth, W., Peters, C., Van de Ven, A., Compact Complex Surfaces, SpringerVerlag, Berlin-Heidelberg, 1984.

[B-R] Burns, D., Rapoport, M., On the Torelli problem for Kählerian K3-surfaces, Ann. Scient. Ec. Norm. Sup., 8 (1975), 235-274.

[Ho $1 \& 2$ ] Horikawa, E., On the periods of Enriques surfaces. I, II, Math. Ann., 234 (1978), 73-88; 235 (1978), 217-246.

[Hut] Hutchinson, J. I., On some birational transformations of the Kummer surface into itself, A.M.S. Bulletin, 7 (1901), 211-217.

[Mor] Morrison, D. R., On K3-surfaces with large Picard number, Invent. Math., 75 (1984), 105-121.

[Mum] Mumford, D., Abelian Varieties, Oxford U. Press, Oxford, 1970.

[Nik 1] Nikulin, V., On Kummer surfaces, Izv. Akad. Nauk. SSSR, 39 (1975) , 278-293; Math. USSR Izvestija, 9 (1975), 261-275.

[Nik 2] Nikulin, V., Integral quadratic bilinear forms and some of their applications, Izv. Akad. Nauk. SSSR, 43, No. 1 (1979), 111-177; Math. USSR Izv., 14, No. 1 (1980), 103-167. 
[P-S] Piateckii-Shapiro, I., Shafarevich, I. R., A Torelli theorem for algebraic surfaces of type K3, Izv. Akad. Nauk. SSSR, 35 (1971), 530-572; Math. USSR Izv., 5 (1971), 547-587.

[S-M] Shioda, T., Mitani, N., Singular abelian surfaces and binary quadratic forms, in "Classification of algebraic varieties and compact complex manifolds", Spr. Lec. Notes, No. 412, 1974.

Department of Mathematics

University of Utah

Salt Lake City

UT 84112, USA 